\title{
Chaotic dynamics from interspike intervals
}

\section{Pavlov, A.N.; Sosnovtseva, Olga V.; Mosekilde, Erik; Anishchenko, V.S.}

\section{Published in:}

Physical Review E. Statistical, Nonlinear, and Soft Matter Physics

Link to article, DOI:

10.1103/PhysRevE.63.036205

Publication date:

2001

Document Version

Publisher's PDF, also known as Version of record

Link back to DTU Orbit

Citation (APA):

Pavlov, A. N., Sosnovtseva, O. V., Mosekilde, E., \& Anishchenko, V. S. (2001). Chaotic dynamics from interspike intervals. Physical Review E. Statistical, Nonlinear, and Soft Matter Physics, 63(3), 036205. https://doi.org/10.1103/PhysRevE.63.036205

\section{General rights}

Copyright and moral rights for the publications made accessible in the public portal are retained by the authors and/or other copyright owners and it is a condition of accessing publications that users recognise and abide by the legal requirements associated with these rights.

- Users may download and print one copy of any publication from the public portal for the purpose of private study or research.

- You may not further distribute the material or use it for any profit-making activity or commercial gain

- You may freely distribute the URL identifying the publication in the public portal 


\title{
Chaotic dynamics from interspike intervals
}

\author{
Alexey N. Pavlov, ${ }^{1}$ Olga V. Sosnovtseva, ${ }^{1}$ Erik Mosekilde, ${ }^{2}$ and Vadim S. Anishchenko ${ }^{1}$ \\ ${ }^{1}$ Nonlinear Dynamics Laboratory, Department of Physics, Saratov State University, Astrakhanskaya Street 83, 410026, Saratov, Russia \\ ${ }^{2}$ Department of Physics, Technical University of Denmark, 2800 Kgs. Lyngby, Denmark
}

(Received 29 June 2000; published 21 February 2001)

\begin{abstract}
Considering two different mathematical models describing chaotic spiking phenomena, namely, an integrate-and-fire and a threshold-crossing model, we discuss the problem of extracting dynamics from interspike intervals (ISIs) and show that the possibilities of computing the largest Lyapunov exponent (LE) from point processes differ between the two models. We also consider the problem of estimating the second LE and the possibility to diagnose hyperchaotic behavior by processing spike trains. Since the second exponent is quite sensitive to the structure of the ISI series, we investigate the problem of its computation.
\end{abstract}

DOI: 10.1103/PhysRevE.63.036205

PACS number(s): 05.45.-a, 87.10.+e

\section{INTRODUCTION}

An important problem in modern science is the study of information processing by biological systems. Optical, chemical, and acoustic signals, in fact all types of information which is received by the sensory system, are encoded by nerve cells into sequences of pulses of similar shape (spikes) before they are transmitted to the brain. Although the molecular mechanisms of this encoding are partly understood, a significant number of questions remain relating to the function of the pulses as a code [1].

A nerve cell can be considered as an example of a threshold device that transforms an input signal $S(t)$ into an output sequence of spikes. The shape of the output pulses does not depend on the external signal, and the information about the structure of $S(t)$ is reflected in the time intervals between successive firings (interspike intervals of the action potential train or ISIs). Such processes are often referred to as point processes. They have been the topic of a large number of studies in the physical literature [2]. Recently, point processes have become of interest from the viewpoint of reconstruction of dynamical systems [3-7]. When applying the reconstruction to a threshold device subject to an external force $S(t)$ the question arises: Can the state of the forcing system be identified from the output ISI sequence? An answer was first provided by Sauer $[3,4]$, who proved that Takens embedding theorem [8] can be extended to the case of point processes. As a consequence, the ISI may be considered as a new state variable that allows us to study the lowdimensional dynamics at the input of a neuron. The possibility to characterize numerically the properties of the external signal when only processing spike train has been tested in a number of works [5-7].

Rather popular and at the same time biologically motivated approaches to the analysis of spiking phenomena are threshold-crossing (TC) and integrate-and-fire (IF) models. Both approaches consider often a chaotic input signal $S(t)$, e.g., a variable of some dynamical system in the case of TC models or its linear transformation for IF models [5].

Within the framework of the first approach, the times $T_{i}$ are determined as the moments when the signal $S(t)$ crosses the level $S=\Theta$ in a particular direction. From the viewpoint of dynamical system theory, this condition sets the equation of a secant plane in phase space. Therefore, the interspike intervals $I_{i}=T_{i+1}-T_{i}$ (TC ISIs) can be interpreted as return times to a Poincaré secant.

Within the framework of IF models a sequence of interspike intervals $I_{i}$ (IF ISIs) can be obtained from the equation

$$
\int_{T_{i}}^{T_{i+1}} S(t) d t=\theta, \quad I_{i}=T_{i+1}-T_{i} .
$$

Here, $\theta$ is a threshold level and $T_{i}$ are the times when the firing threshold is reached and spikes occur. After such events the value of the integral is reset to zero.

In our previous works $[9,10]$, the possibility of extracting dynamics from return times was tested numerically. It was shown that the largest Lyapunov exponent (LE) $\lambda_{1}$ that characterizes the chaotic dynamics of the signal can be extracted from TC ISIs if the mean value of the return time does not exceed some temporal scale. In the present study we show that the possibilities of extracting dynamics from interspike intervals differ between the above-mentioned models. We also discuss the problem of computing the second Lyapunov exponent from ISIs and, hence, the possibility of diagnosing hyperchaotic signals by processing spike trains.

\section{COMPUTING THE LARGEST LE FROM ISI SERIES}

Let us first discuss the TC model. A useful approach to reconstruct the metrical and dynamical properties of a lowdimensional signal $S(t)$ from return times has been proposed by Janson et al. [9]. It is based on the notion of an instantaneous frequency for chaotic oscillations. Briefly speaking, we have suggested to analyze the temporal dependence of the average instantaneous frequency that maintains the characteristics of the chaotic attractor and can be obtained to some approximation from TC ISIs [9]. 

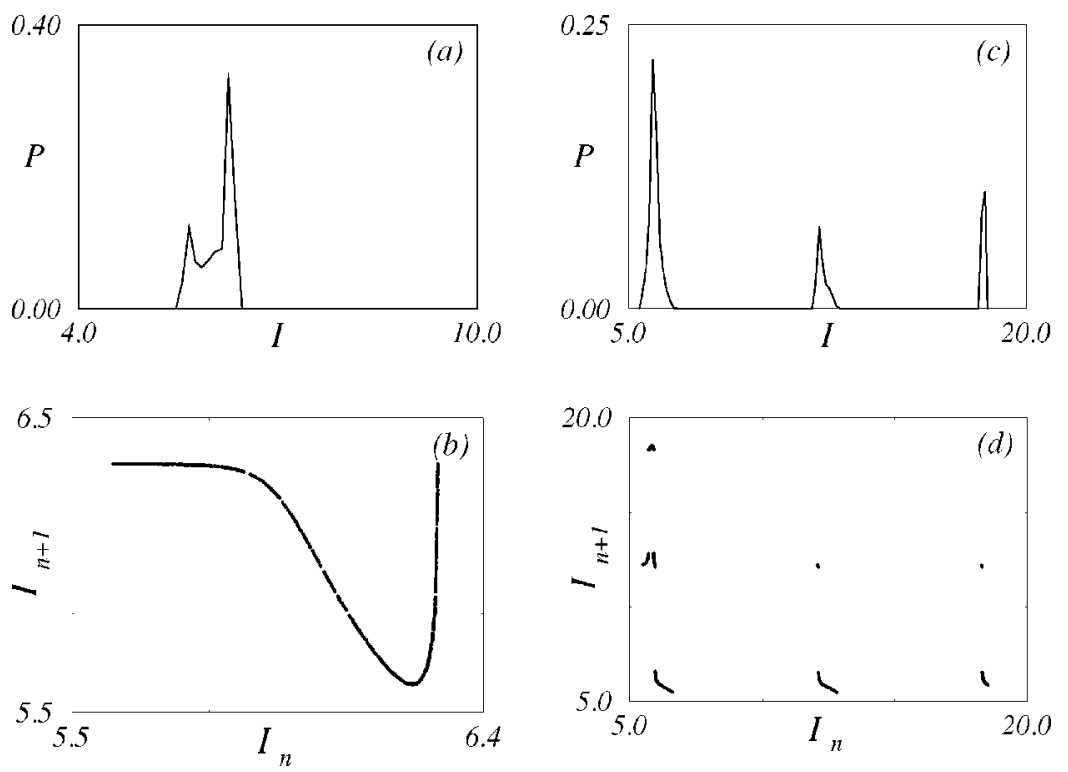

FIG. 1. Probability distributions $P(I)$ of TC ISIs and return time maps appropriate to $\Theta=0$ (a,b) and $\Theta=11(\mathrm{c}, \mathrm{d})$, respectively. As $\Theta$ is shifted away from zero certain loops cease to intersect the Poincaré secant, and contributions to $P(I)$ with longer time intervals arise.
Let us consider the Rössler system

$$
\begin{gathered}
\frac{d x}{d t}=-(y+z), \\
\frac{d y}{d t}=x+0.15 y, \\
\frac{d z}{d t}=0.2+z(x-10) .
\end{gathered}
$$

Here, $S(t)=x(t)$, and the secant plane is specified as $x=0$. A projection of the chaotic attractor on the plane $(x, y)$ resembles a smeared limit cycle [11]. Using the Poincare section, to each rotation (i.e., to each TC ISI) we can attribute a phase increase of $2 \pi$ [11]:

$$
\varphi(t)=2 \pi \frac{t-T_{i}}{I_{i}}+2 \pi i, \quad T_{i} \leqslant t<T_{i+1} .
$$

We may hereafter introduce the points $\omega\left(T_{i}\right)=2 \pi / I_{i}$ that represent the values of the average instantaneous frequency during a return time $I_{i}$.

From the viewpoint of reconstruction it is preferable to analyze data with constant time interval $\Delta t$. For this purpose the points $\omega\left(T_{i}\right)$ can be interpolated by a smooth function (e.g., by a cubic spline) $\omega_{\text {int }}(t)$. Janson et al. [9] showed that $\omega_{\text {int }}(t)$ in many cases allows us to estimate the properties of chaotic oscillations $x(t)$ with good accuracy.

If the value of the threshold level $\Theta$ differs from zero, the situation becomes more complicated. An increase of $\Theta$ changes the structures of the TC ISI series and return time map, because certain loops cease to intersect the secant plane (Fig. 1). Shifting of the firing threshold has a familiar interpretation: Suppose that we change the amplitude or the mean value of the input signal $S(t)$ at constant $\Theta>0$. In the framework of dynamical systems theory the properties of the forcing system (e.g., the dimension and LE) are invariant to linear transformations of the coordinates. The question is as follows: Under what conditions is it possible to extract numerically the characteristics of the chaotic oscillations $S(t)$ at the input of the threshold device from the output signal (i.e., from the TC ISI-sequence)? In a previous study [10] we have shown that the largest LE can be computed from return times if the mean value $\bar{I}$ of the TC ISI is less than some temporal scale $T_{c}$ (Fig. 2) that can be taken approximately to be equal to the prediction time (or Lyapunov time) for the chaotic oscillations [12].

Moreover, even if some loops of the phase-space trajectory do not cross the plane $S=\Theta$, the value of the largest LE can be obtained almost with the same accuracy as in the case
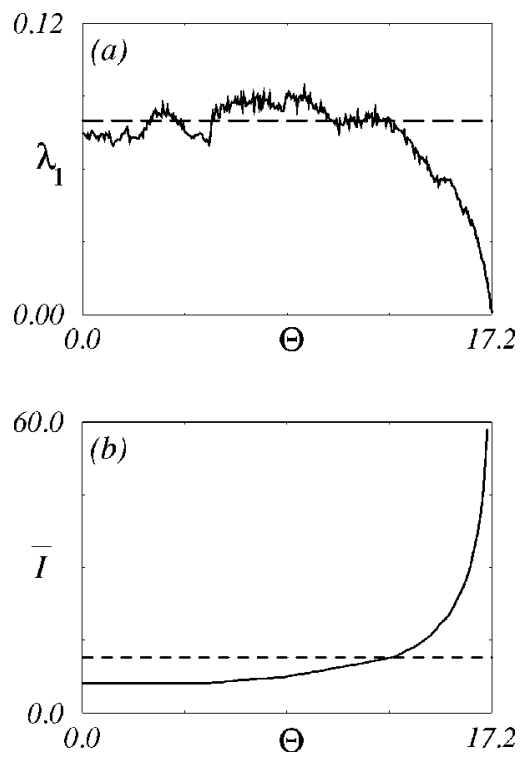

FIG. 2. (a) Dependence of the largest LE $\left(\lambda_{1}\right)$ estimated from TC ISIs using the method [13]; (b) corresponding values of the mean ISI $\bar{I}$ vs the firing threshold $\Theta$. The first coordinate of Eqs. (2) has been used as the input signal $S(t)=x(t)$. The largest LE remains nearly independent of $\Theta$ as long as $\bar{I}$ falls below a certain threshold. 

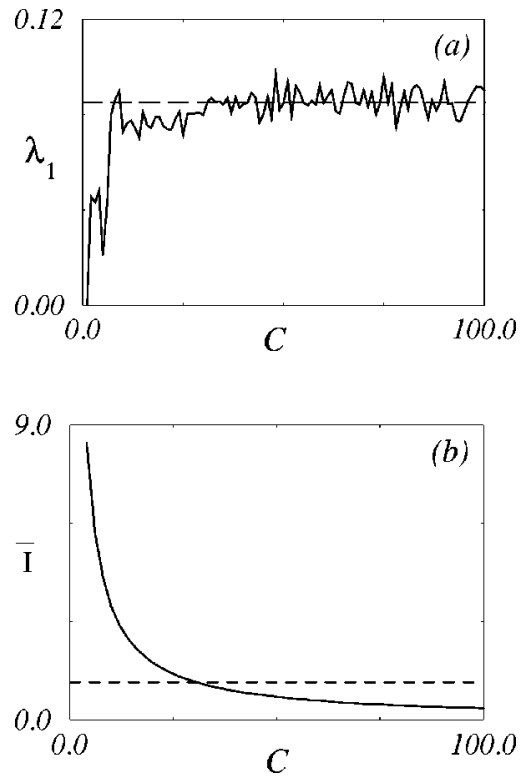

FIG. 3. Dependence of the largest LE estimated from IF ISIs using the method [13] (a) and corresponding values of the mean ISI vs the shift $C$ of the chaotic signal (b). The value of threshold level $\theta=35$.

of small thresholds (where crossing of the secant plane occurs during each rotation). Although the metrical properties are rather sensitive to the choice of $\Theta$ and often cannot be estimated [5], we can still extract dynamics from the interspike intervals.

Now consider the IF model. Following Racicot and Longtin [6], at high firing rates an ISI represents a nonlinear transformation of the external signal

$$
I_{i} \approx \theta / S\left(T_{i}\right) \text {. }
$$

Therefore, we can write

$$
\frac{1}{I_{i}} \approx \frac{1}{\theta} S\left(T_{i}\right)=k S\left(T_{i}\right) .
$$

Equation (5) implies that at fixed moments $T_{i}$ we know the linear transformation of the input signal. The points $1 / I_{i}\left(T_{i}\right)$ are again interpolated by a smooth function $S_{\text {int }}(t)$ that can then be used in the reconstruction. Since $S_{\text {int }}(t) \approx k S(t)$ we expect that the interpolated temporal dependence maintains both the metrical and the dynamical properties of the attractor corresponding to the chaotic forcing. We have carried out such a calculation taking input signal $S(t)=x(t)+C$, where $x(t)$ is the first coordinate of the Rössler system (2) and $C$ is a constant shift ( $C=40, \theta=35$ ) [6]. The longer $\theta$ is, the less is the accuracy of Eq. (4). Aiming to investigate how the quality of the reconstruction of the attractor's characteristics depends on the mean value $\bar{I}$ of the IF ISIs, we can change the constant $C$ at fixed $\theta$ instead of shifting the firing threshold as for TC model. An increase of $C$ leads an increase of the firing rate and a decrease of $\bar{I}$ (Fig. 3). It is clearly seen that the value of $\lambda_{1}$ does not change significantly as long as

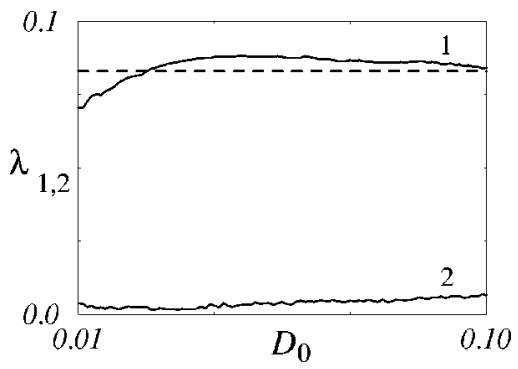

FIG. 4. Results of computing two LEs from TC ISI series obtained with $\Theta=0$ vs one of the parameters from the algorithm [13]. $D_{0}$ is the initial separation between phase trajectories in the restored attractor. First, a renormalization of the reconstructed phase portrait to the range $[0,1]$ has been performed.

$C \geqslant 30$. This corresponds to $\bar{I}<T_{0} / 4$, where $T_{0}$ is the basic period of the chaotic oscillations $x(t)$. For small $C$ the length of time window spanned by a reconstructed state vector, becomes quite large that leads to the effect of attractor degrading [5].

We conclude that the largest LE of the driving signal can be extracted from the output sequence of interspike intervals for TC as well as for IF models if the mean value of the ISI does not exceed some temporal scale $T_{c}$. This scale takes different values for each type of mathematical model used to describe the spiking phenomena: $T_{c}$ is less than $T_{0} / 4$ for IF models (for signals with clearly expressed basic period) and does not exceed the Lyapunov time for TC models. The larger value of the characteristic scale for return times in the latter case is presumably connected with slower temporal variations for the average instantaneous frequency $\omega_{\text {int }}(t)$ in comparison with the oscillations of $S_{\text {int }}(t)$.

\section{COMPUTING TWO LE FROM RETURN TIMES}

Let us now consider a more complicated problem, namely, the extraction of the second $\operatorname{LE~}\left(\lambda_{2}\right)$ from point processes. Choose again the first coordinate of the Rössler system (2) as the input signal $S(t)$ for the TC-model. Figure 4 shows the results for two LE computed with $\Theta=0$ when the TC ISI sequence has only one characteristic temporal scale. The quality of extracting dynamics can be considered as fairly good ( $\lambda_{2}$ is close to zero).

This situation is changed dramatically if there exist several scales in the return times [Figs. $1(\mathrm{c}, \mathrm{d})]$. In this case, the second LE takes nonzero values, and the corresponding regime of the external drive $S(t)$ can be wrongly diagnosed as hyperchaotic. The reason is that unlike the case of small thresholds, an attractor restored from TC ISIs for large $\Theta$ is highly inhomogeneous [10].

A possible way of solving the problem of inhomogeneity for chaotic processes with a clearly expressed basic frequency consists in the generalization of the procedure for instantaneous phase definition. To each crossing of the secant plane, we can attribute a $2 \pi k_{i}$ phase increase: 


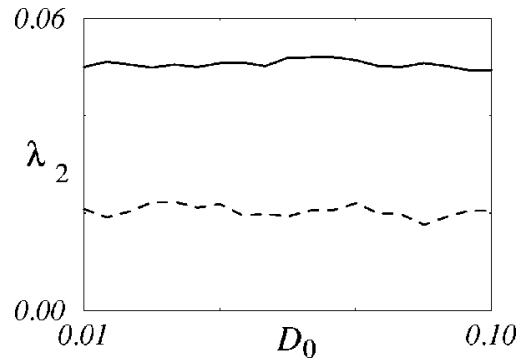

FIG. 5. Results of computing the second LE from TC ISI series appropriate to $\Theta=11$ vs the initial distance between phase trajectories. Solid and dashed lines correspond to different procedures for the introduction of the instantaneous phase: via Eq. (3) and via Eq. (6), respectively. In both cases, the error in the estimation of $\lambda_{2}$ is quite large.

$$
\varphi^{*}(t)=2 \pi k_{i} \frac{t-T_{i}}{I_{i}}+2 \pi \sum_{j=1}^{i} k_{j}, \quad T_{i} \leqslant t<T_{i+1}
$$

where $k_{i}$ are no longer constant, but can be obtained from the condition of slow changes of the average instantaneous frequency $\omega^{*}\left(T_{i}\right)=2 \pi k_{i} / I_{i}$ for successive TC ISIs. The points $\omega^{*}\left(T_{i}\right)$ are interpolated by a smooth function $\omega_{i n t}^{*}(t)$ used further in the attractor restoration. Such an approach solves the problem of inhomogeneity, but in our experiments with large $\Theta$ we were unable to estimate $\lambda_{2}$ in both cases [for $\omega_{\text {int }}(t)$ as well as for $\left.\omega_{i n t}^{*}(t)\right]$, see Fig. 5. Therefore, we conclude that the second LE, unlike the largest exponent, is relatively sensitive to the choice of the firing threshold, and our abilities to extract $\lambda_{2}$ are restricted to the case when threshold-crossing occurs during each rotation of the phasespace trajectory.

In the following we shall discuss only the case of small $\Theta$. Since the quality of extracting dynamics is quite good for such threshold levels (Fig. 4), it becomes relevant to ask: Is it possible to diagnose a hyperchaotic regime when processing spike trains? To answer this question consider two coupled Rössler systems as described in [14]:

$$
\begin{gathered}
\frac{d x_{1}}{d t}=-\omega_{1} y_{1}-z_{1}+c\left(x_{2}-x_{1}\right), \\
\frac{d y_{1}}{d t}=\omega_{1} x_{1}+\alpha y_{1}, \\
\frac{d z_{1}}{d t}=\beta+z_{1}\left(x_{1}-\mu\right),
\end{gathered}
$$

$$
\begin{gathered}
\frac{d x_{2}}{d t}=-\omega_{2} y_{2}-z_{2}+c\left(x_{1}-x_{2}\right), \\
\frac{d y_{2}}{d t}=\omega_{2} x_{2}+\alpha y_{2}, \\
\frac{d z_{2}}{d t}=\beta+z_{2}\left(x_{2}-\mu\right),
\end{gathered}
$$

where the parameters $\alpha, \beta$, and $\mu$ govern the dynamics of each subsystem. $c$ is the coupling parameter, $\omega_{1}=\omega_{0}+\Delta$, and $\omega_{2}=\omega_{0}-\Delta$ are the basic frequencies and $\Delta$ is the mismatch between them. The parameters are chosen as follows [14]: $\alpha=0.15, \beta=0.2, c=0.02, \Delta=0.0093$, and $\omega_{0}=1.0$.

The dependence of the two largest LE for a hyperchaotic regime versus $\mu$ as calculated from Eqs. (7) is shown in Fig. 6(a). Figure 6(b) represents the results of extracting dynamics from TC ISI series recorded as time intervals between successive crossings of the plane $x_{1}=0$ (black points), i.e., when the Poincaré secant is introduced for the first subsystem. We can see, that only the dependence 1 of the largest LE in Fig. 6(a) can be estimated from the analysis of return times. This situation is also observed when considering $y_{1}(t)$ as the forcing signal acting on the TC model [Fig. 6(b), white points]. By analogy, we can set the equation of the Poincare secant for the other subsystem. Figure 6(c) show the corresponding results for the series of times between successive returns to the plane $x_{2}=0$ (black points) and $y_{2}=0$ (white points) by the phase-space trajectory. When processing the
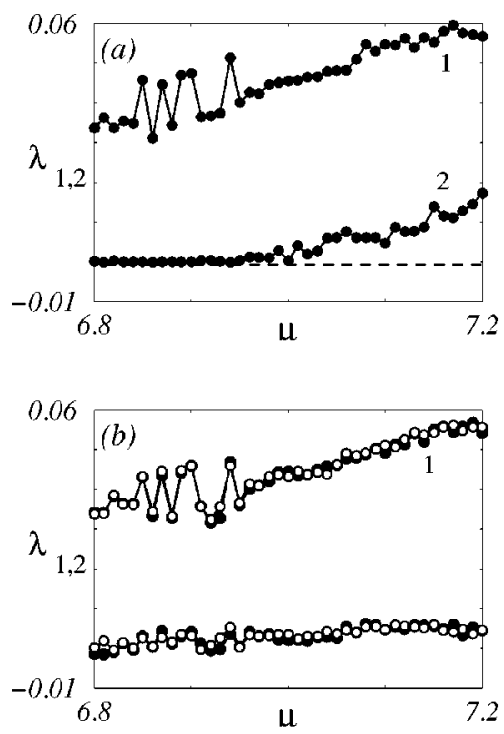
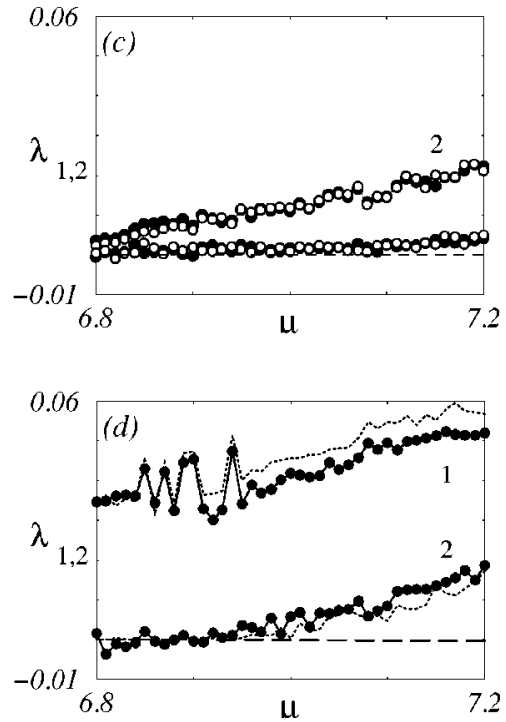

FIG. 6. Computing two LEs from ISI series for a hyperchaotic regime: (a) The results of an estimation $\lambda_{1,2}$ using the approach [15] considered as "true" values; (b) Computing $\lambda_{1,2}$ from return times recorded as time intervals between successive intersections of the plane $x_{1}=0$ (black points) or $y_{1}=0$ (white points); (c) The same as in (b) but for the planes $x_{2}=0$ (or $y_{2}=0$ ); (d) Computing $\lambda_{1,2}$ from two TC ISI sequences simultaneously (taking $x_{1}=0$ and $x_{2}=0$ ). Dotted curves mark "true" values. 
coordinates of the second subsystem, the dependence $\mathbf{2}$ in Fig. 6(a) is reproduced. Thus, we have estimated each positive LE separately: the first positive exponent is obtained if the coordinate of the first subsystem is considered as the external drive for the TC model, and the second positive LE when using the coordinate of the second subsystem of Eqs. (7). These results show that considering a single spike train as the output of the TC model with hyperchaotic forcing is not enough to quantify the input hyperchaotic regime fully.

These results apply if time intervals between intersections of a single plane $x_{j}=0$ (or $y_{j}=0$ ) are used in the attractor restoration. When the opportunity exists to measure return times for each of the coupled dynamical systems (of course, this may be impossible in real experiments), then the properties of the complex processes can be quantified more precisely [Fig. 6(d)].

\section{SUMMARY}

The main results of this study are the following. The largest LE of a chaotic regime with one-dimensional projection acting as forcing signal on IF or TC models can be extracted from an output sequence of interspike intervals if the mean ISI does not exceed some temporal scale $T_{c}$. These scales differ for each of the mathematical approaches describing spiking phenomena: for IF models $T_{c}$ is less than the time of corresponding the first zero value of the correlation function. For TC model the typical scale is significantly larger and approximately equals the prediction time. In this work, the results for Rössler systems have been presented. Subsequent experiments using other systems justify the obtained conclu- sions. Therefore, the largest LE estimated from ISI series is maintained under linear transformations of the chaotic input signal. Moreover, the precision of extracting dynamics does not depend on the structure of ISIs under the formulated conditions. Note, that we speak about the numerical estimation of attractor characteristics. In practice, when processing a finite amount of data, the problem of the quality of reconstruction is important $[5,6]$ although well-known theoretical results [4] have no limitations on the mean value of ISIs.

The second LE is more sensitive to the structure of the output process and can be extracted from return times only if all loops of the phase-space trajectory cross the secant plane.

The knowledge of TC ISIs measured when the onedimensional projection of the hyperchaotic signal in the coupled system acts as input to the TC model is not enough to diagnose a hyperchaotic behavior, and measuring return times from each subsystem is necessary for this purpose.

We have shown that computing two LEs can lead to incorrect conclusions: the chaotic regime with several temporal scales can be diagnosed as hyperchaotic (Fig. 5). At the same time, complex dynamics with two positive exponents may be not quantified [Figs. 6(b,c)].

\section{ACKNOWLEDGMENTS}

A.P. acknowledges INTAS (Grant No. YSF 99-4050) and O.S. acknowledges support from the Danish Research Academy. The research described in this publication was made possible in part by Award No. REC-006 of the U.S. Civilian Research \& Development Foundation for the Independent States of the Former Soviet Union (CRDF).
[1] S.P. Strong, R. Koberle, R.R. de Ruyter van Steveninck, and W. Bialek, Phys. Rev. Lett. 80, 197 (1998); H. C. Tuckwell, Introduction to Theoretical Neurobiology (Cambridge University Press, Cambridge, 1988), Vols. 1 and 2.

[2] A. Longtin, A. Bulsara, and F. Moss, Phys. Rev. Lett. 67, 656 (1991); A. Garfinkel, M.L. Spano, W.L. Ditto, and J.W. Weiss, Science 257, 1230 (1992); S.J. Schiff, K. Jerger, D.H. Duong, T. Chang, M.L. Spano, and W.L. Ditto, Nature (London) 370, 615 (1994); F. Moss, and X. Pei, ibid. 376, 211 (1995); K.A. Richardson, T.T. Imhoff, P. Grigg, and J.J. Collins, Phys. Rev. Lett. 80, 2485 (1998); D. Christini, and J.J. Collins, ibid. 75, 2782 (1995); X. Pei and F. Moss, Nature (London) 379, 618 (1996); P. So, E. Ott, S. Schiff, D. Kaplan, T. Sauer, and C. Grebogi, Phys. Rev. Lett. 76, 4705 (1996).

[3] T. Sauer et al., Phys. Rev. Lett. 72, 3911 (1994).

[4] T. Sauer, in Nonlinear Dynamics and Time Series, edited by C. Culter and D. Kaplan, Fields Institute Communications Vol. 11 (American Mathematical Society, Providence, RI, 1997), p. 63.

[5] R. Castro and T. Sauer, Phys. Rev. E 55, 287 (1997).

[6] D.M. Racicot and A. Longtin, Physica D 104, 184 (1997).

[7] R. Hegger and H. Kantz, Europhys. Lett. 38, 267 (1997); M. Ding and W. Yang, Phys. Rev. E 55, 2397 (1997); R. Castro and T. Sauer, Phys. Rev. Lett. 79, 1030 (1997); Phys. Rev. E 59, 2911 (1999).

[8] F. Takens, in Dynamical Systems and Turbulence, edited by D. A. Rang and L. S. Young, Lecture Notes in Mathematics Vol. 898 (Springer-Verlag, Berlin, 1981), p. 366.

[9] N.B. Janson, A.N. Pavlov, A.B. Neiman, and V.S. Anishchenko, Phys. Rev. E 58, R4 (1998).

[10] A.N. Pavlov, O.V. Sosnovtseva, E. Mosekilde, and V.S. Anishchenko, Phys. Rev. E 61, 5033 (2000).

[11] A.S. Pikovsky, M.G. Rosenblum, G.V. Osipov, and J. Kurths, Physica D 104, 219 (1997).

[12] J.D. Farmer and J.J. Sidorowich, Phys. Rev. Lett. 59, 845 (1987); J. Brindley and T. Kapitaniak, in Chaotic, Fractal and Nonlinear Signal Processing, edited by R. A. Katz, AIP Conf. Proc. No. 375 (AIP Press, Woodbury, New York, 1996), p. 605.

[13] A. Wolf, J.B. Swift, H.L. Swinney, and J.A. Vastano, Physica D 16, 285 (1985).

[14] D.E. Postnov, T.E. Vadivasova, O.V. Sosnovtseva, A.G. Balanov, V.S. Anishchenko, and E. Mosekilde, Chaos 9, 227 (1999).

[15] G. Benettin, L. Galgani, A. Giorgilli, and J.M. Strelcyn, Meccanica 15, 9 (1980). 